\title{
A Practical Approach to Filtering Facepiece Respirator Decontamination and Reuse: Ultraviolet Germicidal Irradiation
}

Mark P. Ridder, $M D^{1}$

Katie D. Paladino, RT $(R)^{2}$

John J. Lowe, $P h D^{3}$

Mark E. Rupp, $M D^{1, *}$

\author{
Address \\ *,1Division of Infectious Disease, University of Nebraska Medical Center, 985400 \\ Nebraska Medical Center, Omaha, NE, 68198-5400, USA \\ Email: merupp@unmc.edu \\ ${ }^{2}$ Department of Enterprise Quality and Outcomes, Nebraska Medicine, Omaha, NE, \\ 68198, USA \\ ${ }^{3}$ Global Center for Health Security, University of Nebraska Medical Center, Omaha, \\ $\mathrm{NE}, 68198, \mathrm{USA}$
}

Published online: 6 April 2021

(C) The Author(s), under exclusive licence to Springer Science+Business Media, LLC, part of Springer Nature 2021

This article is part of the Topical Collection on New Technologies and Advances in Infection Prevention

Keywords Filtering facepiece respirator - Decontamination and reuse $\cdot$ Ultraviolet germicidal irradiation -

Microwave-generated steam $\cdot$ Warm moist heat $\cdot$ Vaporized hydrogen peroxide

\begin{abstract}
Purpose of review Prior outbreaks of respiratory viruses have demonstrated the need for adequate personal protective equipment (PPE) for healthcare workers, particularly filtering facepiece respirators (FFR). Due to shortfalls of PPE during the SARS CoV-2 pandemic, the need for FFR decontamination and reuse (FFR-DR) strategies is paramount. This paper aims to discuss primary decontamination strategies, with an in-depth analysis of ultraviolet germicidal irradiation (UVGI), arriving at the decontamination strategy utilized at the Nebraska Medical Center (NMC).

Methods Review of the primary literature in regard to FFR-DR as well as a synopsis of the current protocol for FFR-DR at NMC.

Recent findings UVGI demonstrates effective decontamination of multiple pathogens-including several human respiratory viruses-while maintaining mask integrity and filtering capacity. UVGI was associated with degradation of strap
\end{abstract}


integrity at higher doses than that utilized for decontamination or with reuse beyond 20 times.

Summary UVGI effectively decontaminates N95 FFRs without significant reduction to fit or strap integrity and can be employed as a strategy for FFR-DR in times of emergency.

Introduction and regulatory considerations

After prior outbreaks of H1N1 influenza, Middle East Respiratory Syndrome (MERS), and Severe Acute Respiratory Syndrome (SARS), respirator shortages, particularly N95 filtering facepiece respirators (FFRs) were predicted for subsequent outbreaks [1]. Unfortunately, maintaining large stockpiles of personal protective equipment (PPE) to meet needs during a pandemic has been regarded as impractical, particularly for one-time use equipment items. Estimates based upon modeling of severe influenza pandemics range from the need of 1.7 to 7.3 billion FFRs, assuming $20-30 \%$ of the population were ill. Despite these predicted needs, there were only an estimated 60 million FFRs available at the time of the onset of the current COVID-19 pandemic [2•].

In addition to administrative and engineering controls, PPE, including N95 FFRs or a higher level of respiratory protection, should be used in the care of patients known or suspected to be infected with SARS-CoV-2, the causative agent of COVID-19. N95 FFRs are designed and manufactured to be used once and then to be disposed. There are no manufacturer-authorized methods for FFR decontamination. However, N95 FFRs remain in critically short supply and the CDC has recommended contingency strategies to expand availability [3•]. Institutions should evaluate and monitor N95 FFR supply and usage and determine whether a crisis strategy is indicated [3•]. Options that should be explored by institutions include use of NIOSH-approved alternatives to N95 FFRs (elastomeric half mask and fullface piece air-purifying respirators, powered air-purifying respirators (PAPRs), and FFR with higher levels of protection (N99, N100, etc.). Furthermore, N95 FFRs can be used beyond the manufacturer's designated shelf life and can be utilized in an extended use manner (e.g., N95 FFR worn for a prolonged period for multiple patient contacts before being discarded). Limited reuse and decontamination of N95 FFRs can also be considered. Under specific short supply circumstances,
OSHA has outlined enforcement discretion to allow for the reuse of N95 FFRs [4] and the FDA has issued emergency use authorization (EUA) to increase the supply of PPE including a process to decontaminate FFRs [5].

In anticipation of a pandemic and the need for rapid and dramatic expansion of N95 FFR supply, work has been done to explore methods for effective, efficient, and feasible decontamination of FFRs $[2 \bullet, 6-9,10 \bullet \bullet$, 11]. For the current pandemic, an effective decontamination method must effectively reduce the burden of SARS-CoV-2 and other respiratory pathogens while not adversely affecting the fit or function of the FFR or resulting in a residual chemical hazard. As of April 2020, the CDC identified ultraviolet germicidal irradiation (UVGI), vaporous hydrogen peroxide, and moist heat as showing the most promise for FFR decontamination. However, it must be realized that there are approximately 500 NIOSH-certified N95 FFR models, each with unique characteristics. A decontamination method appropriate for one N95 FFR model may not be effective for another. Although there are limited data to support multiple decontamination and donning/doffing cycles, NIOSH recommends limiting reuse to five cycles [12]. Clearly, regardless of the number of decontamination cycles, FFRs should be discarded when they are visibly contaminated with blood or respiratory secretions or they are damaged, malformed, or unable to pass fit testing or seal checking. Finally, FFR decontamination and reuse should cease as soon as adequate supplies of FFRs can be obtained to meet the projected needs of the organization. At such time, FFRs should be used per manufacturer recommendations and be used as singleuse items. Organizations wishing to use respirators that are designed for reuse should investigate the introduction of elastomeric respirators and PAPRs.

In evaluating potential methods for N95 FFR reprocessing, several questions need to be answered in order to develop protocols for adoption by healthcare facilities: 
1.) Does the decontamination technique eradicate the target virus? Will the proposed technique(s) work across different pathogens?

2.) Does the proposed process reduce the efficacy of filtration or respirator fit with subsequent uses?

3.) Is the process scalable for most healthcare institutions with reasonable throughput and low cost?

4.) Will healthcare workers accept and use decontaminated respirators? What barriers to implementation may they face?

With these issues in mind, this paper will proceed through a discussion on 3 major decontamination techniques: warm moist heat (WMH) including microwave-generated steam (MGS), vaporous hydrogen peroxide, and ultraviolet germicidal irradiation (UVGI) (Table 1).

At the Nebraska Medical Center, the primary technique for respirator decontamination has been UVGI owing to its low upfront cost, robust virologic effect, estimated respirator preservation of ten cycles, and response to prior feedback from healthcare workers [13]. Therefore, after a brief discussion of various decontamination strategies, this paper will focus primarily on UVGI.

\section{An overview of different decontamination strategies}

\section{Moist heat}

Moist heat $(\mathrm{MH})$ decontamination is a relatively straight-forward process which is usually performed by one of two modalities. The first is the production of $\mathrm{MH}$ by means of a conventional sealed oven, which is similar to autoclave decontamination. In general, water is placed in a sealed oven along with the contaminated respirator and heated to a target temperature of approximately $70{ }^{\circ} \mathrm{C}$ $\left(160^{\circ} \mathrm{F}\right)$. A typical cycle time is approximately $30 \mathrm{~min}$. General results with this process are favorable, with $\log _{10}$ reductions of influenza virus greater than 4.0. This process can be easily replicated, but is generally difficult to scale to larger volumes [7]. The other modality for the production of $\mathrm{MH}$ is microwavegenerated steam (MGS), which is a process involving an industrial-grade microwave oven and magnetrons. The magnetrons are used with rotation for the

Table 1. Overview of methods for FFR-DR

\begin{tabular}{|c|c|c|}
\hline Method & Benefits & Drawbacks \\
\hline $\begin{array}{l}\text { Moist heat } \\
\text { 1. Conventional oven generated moist heat }\end{array}$ & $\begin{array}{l}\circ \text { Effective } \\
\circ \text { Equipment readily on hand }\end{array}$ & $\begin{array}{l}\text { - Long sterilization cycles } \\
\text { o Limited space }\end{array}$ \\
\hline 1. Microwave-generated moist heat & $\begin{array}{l}\text { - Effective } \\
\circ \text { Fast cycle time }\end{array}$ & $\begin{array}{l}\text { o Requires specialized equipment } \\
\text { o Labor intensive } \\
\text { o Masks with metal can melt }\end{array}$ \\
\hline Vaporous hydrogen peroxide & $\begin{array}{l}\circ \text { Effective } \\
\circ \text { Gentle on mask materials }\end{array}$ & $\begin{array}{l}\text { - Difficult to scale } \\
\text { - Requires specific equipment } \\
\text { - Cycles can abort unexpectedly }\end{array}$ \\
\hline Ultraviolet germicidal irradiation & $\begin{array}{l}\text { Effective } \\
\text { o Scalable } \\
\text { - Equipment readily on hand } \\
\text { - Fast cycle times } \\
\text { - Less labor intensive }\end{array}$ & $\begin{array}{l}\text { - Requires exposure of all surfaces (shadowing) } \\
\text { - Reduces strap integrity over time } \\
\text { o Limited specific FFR types }\end{array}$ \\
\hline
\end{tabular}


purpose of even distribution of microwaves. Sterilizers have a water reservoir located below the target FFR, which then in turn produces sterilizing steam to decontaminate the respirator. The FFR is usually placed on a rack in the steam sterilizer to better ensure exposure of all surfaces of the FFR. Generally, microwave cycle run times are between 60 to $90 \mathrm{~s}$ [8]. It should be noted that microwave decontamination can also be performed without the use of steam, but requires $90 \mathrm{~min}$ in order to attain similar results [8]. Key considerations with MGS are that it can provide better through-put time compared to other methods. However, loading masks onto sterilizers is labor intensive. Nonmicrowave-based methods for generation of moist heat often have associated odors which can be overcome with use of essential oils during the decontamination process [8]. Additionally, MGS is limited by the availability of industrial-grade microwaves which are generally not already available at most healthcare institutions. Furthermore, microwave use can result in arcing if the FFRs contain metal (e.g., moldable nosepiece, strap clips) and it has been noted that some FFRs have melted when this method of decontamination has been employed [8].

Vaporous hydrogen peroxide decontamination is the process of placing the target in a sealed unit, which is then flooded with vaporized hydrogen peroxide. The process has been shown to be an efficacious method of decontamination with adequate $\log _{10}$ reductions of a broad spectrum of microbes without degradation of filtration performance $[9,14]$. Several commercially available vaporous hydrogen peroxide (VHP) systems are marketed in the United States including Bioquell (Ecolab), Steris Life Sciences, and Sterrad (Advanced Sterilization Products). Some degradation of strap elasticity and filtration performance have been noted after repetitive decontamination [15]. Other challenges noted with this method include need for expensive and specialized equipment, space limitations, and cycle times. Another issue with this method is the absorption of hydrogen peroxide vapor into cellulose-based products in masks, resulting in decontamination cycle abortion due to low vapor levels [9]. Overall, this is a promising method of FFR decontamination with certain systems appearing to perhaps be more compatible than others.

Ultraviolet Germicidal Irradiation is the process of exposing the target object for decontamination to ultraviolet light with the dose of irradiation being a function of light intensity and time. Prior studies have demonstrated this method to be an effective means of decontamination, with a greater than $4 \log _{10}$ reduction for various viruses $[2 \bullet, 7,10 \bullet \bullet, 13,16]$. This method of decontamination can be accomplished using devices that many hospitals already use for room decontamination or other purposes (isolation hoods, ventilation decontamination, etc). In addition, it carries the benefit of being able to scale up to allow many target masks to be decontaminated at one time. Issues for this method include the production of off-odors after cycling (which were not found to contain meaningful levels of harmful compounds $[10 \bullet \bullet])$, shadowing effects, and potential degradation of strap function and filtration performance. 
A number of other decontamination methods have been considered and are generally regarded as unsuitable. Autoclaving and use of disinfectant wipes (70\% isopropyl alcohol, hypochlorite, benzalkonium chloride) are not recommended as they are likely to alter FFR performance [6, 11, 17]. Although ethylene oxide does not appear to alter FFR performance, its use is not recommended due to issues related to off-gassing and throughput.

\section{An overview of the N95 FFR UVGI decontamination literature}

As noted, UVGI had strong supporting data for efficacy as a germicidal decontamination process, lower upfront costs, and high throughput volume capacity. As a result, it was employed as a method for FFR decontamination in response to the crisis capacity need due to FFR shortages in the midst of the COVID-19 outbreak. Below, data is reviewed which supplied answers to the questions stated in the introduction. These studies were conducted primarily with the use of H1N1 virus inoculating a variety of different FFRs $[2 \bullet, 10 \bullet \bullet, 16]$.

Radiation dosage

Use of UV radiation for decontamination was extensively tested in the wake of

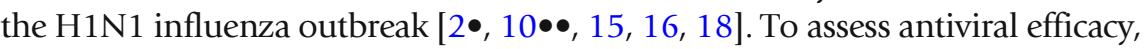
contaminated coupons of $3 \mathrm{M} 1870$ N95 masks were exposed to variable amounts of UV radiation, as measured in $\mathrm{J} / \mathrm{cm}^{2}$. To emulate human use, masks were exposed to soiling agents including synthetic skin oil as well as artificial saliva. One group of coupons was inoculated with virus alone, and another group was inoculated with virus and soiling agents. It was found that viable virus was recovered from samples exposed to $0.5 \mathrm{~J} / \mathrm{cm}^{2}$, but after increasing to

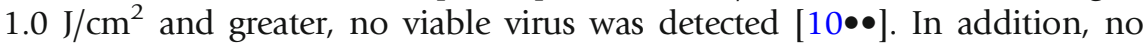
further discernable log difference in viral recovery was noted as UV exposure increased from 1 to $2 \mathrm{~J} / \mathrm{cm}^{2}[10 \bullet \bullet]$. Literature review included several studies with interventions using $1.2 \mathrm{~J} / \mathrm{cm}^{2}$ to $9.5 \mathrm{j} / \mathrm{cm}^{2}$ finding similar germicidal activity with a $\log _{10}$ reduction average of 3.74 [18].

FFR model

Investigators analyzed whether the irradiation dose could be applied universally to FFRs, or if there would be specific limitations to certain brands. Selected models were $3 \mathrm{M}$ 1870, 3M 1960, Kimberly-Clark PFR, Moldex 1512, Precept 65-3395, Gerson 1730, Sperian H-NB095, U.S. Safety AD2N95A, Moldex 1712, U.S. Safety AD4N95, 3M VFlex 1805, Alpha Protech 695, Prestige Ameritech RP 88020, Sperian HC-NB 295F,

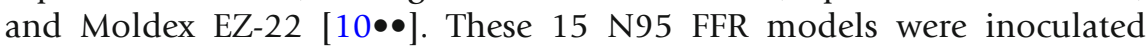
with influenza and exposed to $1 \mathrm{~J} / \mathrm{cm}^{2}$ of irradiation. After decontamination doses were administered, the masks were then assayed for remaining viable virus. Tight environmental controls were required to maintain precision of irradiation and to monitor temperature which tends to increase with increasing applied dosage and can have an

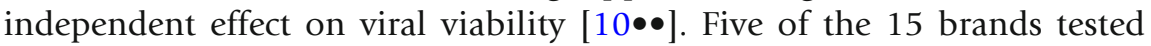
exhibited at least a $3 \log _{10}$ reduction of viable virus. These were the $3 \mathrm{M}$ 1870, 3M 1860, Kimberly-Clark PFR, Moldex 1512, and Moldex 1712 
$[10 \bullet \bullet]$. It was concluded that UVGI can be effective, but it is notably dependent upon environmental factors, as well as the composition of the FFRs. These differences can significantly impact the efficacy of decontamination.

FFR strap integrity

Prior studies have demonstrated that repeated donning and doffing, as well as prolonged FFR use, can have a deleterious effect on fit $[8,9,10 \bullet \bullet, 11-21]$. It is noted that strap integrity degrades over time resulting in FFR strap failure and breakage in some instances. In addition, there are concerns in regard to the maintenance of an adequate fit after repeated strap stretch. In prior studies, it was noted that there is a dose-dependent relationship between the force required for strap breakage and UVGI dose [16, 18]. Given these concerns, special care was taken with an analysis of fit and strap integrity over repeated use as well as fit after UVGI decontamination. All 15 of the above FFRs were tested with original fit as a control; another group with stretching the straps alone for 10 cycles; and then a third group tested with stretching and UVGI for 10 cycles $[10 \bullet \bullet]$. The FFRs were then tested to ascertain reduction of elasticity based upon

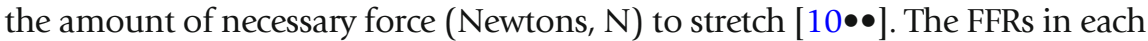
group were then tested for fit by being attached to a breathing simulator with a

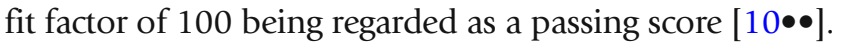

In synopsis, a clear correlation was identified between repeated stretches and reduction in the amount of force needed to stretch FFR straps, which was noted at around 6 strap stretches. After this point, there was not significantly reduced mean peak force required to stretch mask straps $[10 \bullet \bullet]$. When comparing all FFRs, considering stretches and UVGI decontamination, it was noted that there was a statistically significant change in mean force required for the following models after 10 cycles: 3M 1870, Alpha ProTech 965, Gerson 1730, KimberlyClark PFR, and the Moldex 1512 [10••]. In further study, 6 masks were selected to undergo testing for 20 cycles. These were $3 \mathrm{M} \mathrm{1860,3M} \mathrm{1870,3M} \mathrm{VFlex} \mathrm{1805,}$ Kimberly-Clark PFR, Moldex 1512, and U.S. Safety AD4N95 [10••]. Unsurprisingly, a reduction of necessary force for strap stretch was observed with repeated donning and doffing. It was noted that among this group there was no statistical difference between the donning-and-doffing-only group and the UVGI-treated group, suggesting that the repeated donning and doffing are the primary drivers

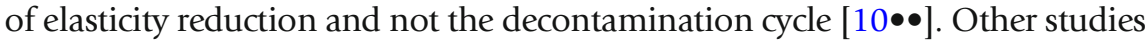
regarding UVGI dose and strap integrity have noted a reduction of strap integrity, with $10-21 \%$ reduction in force required to break straps at an approximate dose of $590 \mathrm{~J} / \mathrm{cm}^{2}$ and $20-51 \%$ reduction at a dose of $2360 \mathrm{~J} / \mathrm{cm}^{2}$ [18] Notably, these radiation doses are far higher than those necessary to achieve decontamination.

Using this information, a logical follow-up question would be how many cycles of decontamination and reuse could be performed before a significant drop-off in strap efficacy was observed. Therefore, these same 6 FFRs models were tested with 0 cycles, 10 cycles, and 20 cycles of UVGI $[10 \bullet \bullet]$. The 3M 1860, 3M 1870, 3M VFlex 1805, Moldex 1512, and U.S. SafetyAD4N95 all had statistically significant reduction of

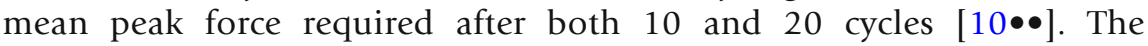
Kimberly-Clark PFR was the only FFR that was unchanged with mean 


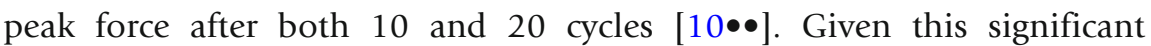
degradation of strap mean peak force for most FFRs with repeated cycles of UVGI and/or donning and doffing, a reasonable conclusion would be that reducing reuse to 10 or fewer cycles would limit FFR failures related to strap elasticity reduction.

\section{FFR fit}

Regardless of strap integrity associated with UVGI dose, the more clinically apt question would be whether UVGI dose affected FFR fit. In order to test this, a quantitative fit test methodology was employed. After repeated donning and doffing alone, as well as with UVGI decontamination for 10 cycles, fit testing was conducted. All of the tested FFRs maintained a fit factor greater than 100 after 10 cycles [10••]. Six masks were then selected to test for 20 cycles as above, once again including the following masks: $3 \mathrm{M}$ 1860, 3M 1870, 3M VFlex 1805, KimberlyClark PFR, Moldex 1512, and U.S. Safety AD4N95. Within this group, it was identified that the 3M 1860, 3M 1870, 3M VFlex 1805, and Moldex 1512 maintained fit factor greater than $100[10 \bullet \bullet]$. These data suggest that the use of UVGI, when used in the context of multiple donning and doffing cycles, does result in some reduction in both strap integrity and fit, but can serve as a viable means for FFR decontamination and reuse if there is proper appreciation for limiting the number of decontamination and don/doff cycles. Healthcare providers should be educated and instructed to discard an FFR if strap elasticity or fit characteristics are in doubt.

\section{FFR filtration and airflow resistance}

In addition, airflow resistance (breathability) has been tested to ensure that UVGI decontamination does not impair flow rates through the FFRs. It is accepted that $25 \mathrm{~mm} \mathrm{H}_{2} \mathrm{O}$ is the maximum exhalation resistance for N95 FFRs [18]. In review of untreated, UV-treated, and don/doff for 10 cycles for 15 N95 FFR models, and 20 don/doff cycles for 3M 1860, 3M 1870, 3M VFLEX 1805, Kimberly-Clark PFR, Moldex 1512, and U.S. Safety AD4N95, testing found that there was reduction of resistance for both the Moldex 1512 and KimberlyClark PFR. However, reduction of resistance is not necessarily an undesirable effect. The rest of the masks did not demonstrate any statistically significant change in airflow resistance $[10 \bullet \bullet$. Minimal change in FFR resistance has been observed in other studies of N95 FFR decontamination [16, 18]. Additionally, the specific FFR models noted above were then tested after 10 and 20 cycles of UVGI decontamination to determine if particle penetration capacity was maintained. In a similar fashion, none of the FFRs demonstrated any significant change and maintained particle penetrance well below 5\%, meeting the intended standard of $95 \%$ particle filtration [10••]. A meta-analysis of 6 papers studying FFR UVGI decontamination noted an average particle penetrance of $1.19 \%$ following decontamination [18]. In addition, peak flow resistance averaged $9.89 \mathrm{mmH} 2 \mathrm{O}$ with none exceeding $11.44 \mathrm{~mm} \mathrm{H20}$, all being sufficiently below the desired target of $25 \mathrm{~mm} \mathrm{H} 20$ [11]. In synopsis, UVGI treatment did not cause a significant reduction in filtration or airflow resistance for tested N95 FFRs. 


\section{Decontamination of different respiratory viruses}

In anticipation of having to use an UVGI decontamination strategy in the event of a novel pathogen outbreak, the $3 \mathrm{M} 1870$ has been tested with various viruses including Influenza types H1N1, H5N1, and 2 different strains of H7N9 as well as Middle Eastern Respiratory Syndrome coronavirus (MERS) as well as Severe Acute Respiratory Syndrome coronavirus (SARS) [10••]. In addition to the viral challenge, the FFRs were also treated with synthetic saliva and sebum. After

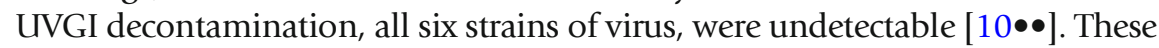
data suggest that UVGI has broad anti-viral activity and is a viable strategy for FFR decontamination and reuse in the event of a respiratory viral outbreak.

\section{Safety of FFRs}

In addition to necessary demonstration of effective decontamination without reduction of fit, strap integrity, filtering capacity, or airflow resistance, the following FFRs have been tested to ensure adequate protection against fluid penetration and flammability after 20 cycles of UVGI: 3M 1860, 3M 1870, 3M VFlex 1805, Kimberly-Clark PFR, Moldex 1512, and U.S. Safety AD4N95 $[10 \bullet \bullet]$. Of the six models tested for flammability, only the Kimberly-Clark

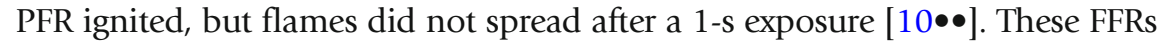
were also tested for fluid penetration with exposure to a high-velocity stream of synthetic blood $[10 \bullet \bullet$. The U.S. FDA allows a failure rate of $4 \%$ in such test conditions. Thirty-two FFRs of each brand were tested allowing for 3 failures. Within testing, 3 brands failed once: 3M 1860, Kimberly-Clark PRF, and Moldex $1512[10 \bullet \bullet$. However, all of the brands were well within the governing criteria

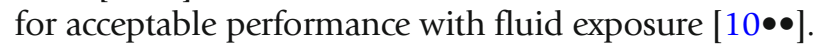

\section{Synthesis of literature review}

The body of literature and extensive work of other investigators, as detailed above, indicates that UVGI at a dose of approximately $1 \mathrm{~J} / \mathrm{cm}^{2}$, is effective at reducing contaminant burden by significant $\log _{10}$ reductions of virus. While in some situations a notable reduction of strap integrity was observed, this did not significantly impair fit factor for these FFRs. Even after multiple cycles of UVGI and reuse, the masks were found to maintain adequate airflow as well as maintaining required particle filtration percentages greater than 95\%. UVGI decontamination showed adequate response across a wide range of viruses suggesting that this strategy could be effective for SARS CoV-2.

\section{Acceptance of UVGI by healthcare providers}

While UVGI FFR-DR can be feasibly, efficiently, and effectively accomplished, whether or not healthcare providers (HCPs) would accept a decontaminated FFR remained in question. As a result, work involving cognitive tasks analysis interviews, focus groups, and surveys were conducted at the following sites: the University of Nebraska Medical Center, (UNMC), Gulf Coast Regional Medical Center (GCRMC) Stony brook University Hospital (SBUH), and University of

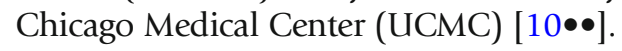

Notable findings from this inquiry $[10 \bullet \bullet]$ included the following:

1. HCPs have strong personal opinions regarding use of FFRs previously worn by others.

2. HCPs have variable training in the use of PPE. 
3. Compliance with FFR is variable, as many are not fit tested regularly.

4. HCPs are concerned if decontamination method(s) are translatable to novel viruses.

5. Practical implications would need thorough planning in order to implement.

6. HCPs would need means to validate adequate decontamination.

Panels of HCPs indicated their belief that, in the context of a pandemic, no FFR would be most unsafe, extended-use FFR would be moderately safer, and FFR with UV decontamination and reuse would be the safest option given in the survey [10••]. In situations with limited FFR availability, the survey indicated that HCPs would likely be amenable to FFR-DR as

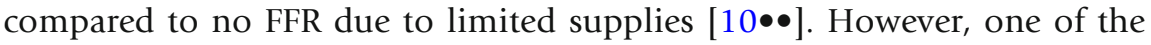
greatest concerns for reuse was the notion of sharing FFRs with other HCPs. Many HCPs felt that in spite of decontamination, they would rather reuse their own decontaminated FFR rather than an FFR from another person $[10 \bullet \bullet$. Surveys also demonstrated that staff would need proper training for FFR use $[10 \bullet \bullet]$. There are concerns that prior to the COVID-19 pandemic, compliance with FFR recommendations, such as annual fit testing was variable $[10 \bullet \bullet$. To better ensure the success of an N95 FFR decontamination and reuse program, integration of education and process

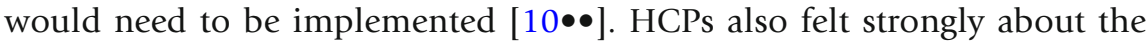
desirability of having the site for FFR decontamination near the point-ofcare. This was driven by concerns of cross-contamination while en route to decontamination, time loss for FFR while in transit, and the needed storage space while waiting for decontamination processing.

\section{The Nebraska Medicine N95 FFR decontamination and reuse experience}

Based on the preceding discussion and the large body of literature and investigative work done by others, Nebraska Medicine developed a protocol for FFR-DR [13]. Since the spring of 2020, due to crisis capacity issues, Nebraska Medicine has utilized a UVGI strategy. The process is described below:

HCPs, employing an extended FFR use protocol, deposit used FFRs, marked with the workers' name, 1st use date, and patient care unit location in brown "dirty" bags after use. These bags, containing the used N95 FFRs, are sent to an onsite decontamination center. At the decontamination center, specially trained UVGI associates, wearing appropriate PPE, remove the contaminated FFRs from the brown bag, gently open the FFR to ensure maximum UV exposure, and hang the FFRs on a clipping line (Fig. 1). Next, the associate exits and, after appropriate doffing and hygiene techniques, initiates the UVGI machine. The exposure in $\mathrm{mJ} / \mathrm{cm}^{2}$ is monitored by sensors and, in general, the decontamination cycle requires 15-20 min [13]. Afterwards, the associate notes the number of decontamination cycles on the FFR and places the decontaminated FFRs in white "clean" paper bags with the workers' 


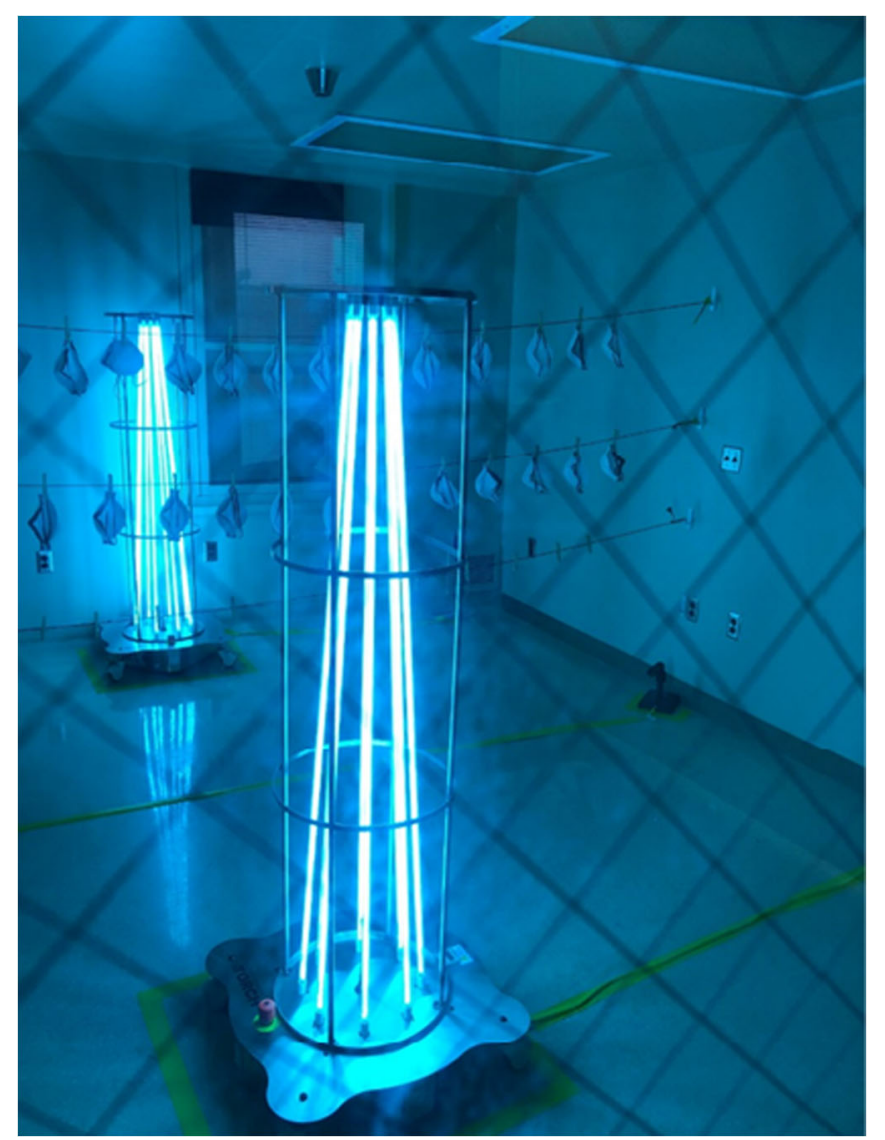

Fig. 1. UVGI lights and hanging lines for N95 FFRs. The walls and ceiling have been painted with UVGI reflective paint to better ensure that all surfaces of the N95 FFRs are exposed to UVGI.

information noted on the outside of the bag. The clean bag is sent back to their corresponding patient care unit for reuse by the individual HCP [13]. HCPs are instructed to discard the FFR if it is soiled, torn, damaged, the straps do not have normal elasticity, or the FFR does not easily achieve a good seal check. Generally, masks are reused approximately 5 to 6 times before being discarded, thus significantly increasing the supply of FFRs compared to single-use FFR scenarios [13].

\section{Discussion}

In the wake of SARS-CoV-2, development of protocols to mitigate FFR shortages have been vital. Multiple decontamination strategies have been explored, and depending upon the current resources of institutions, some may be viable options. Warm moist heat, vaporized hydrogen peroxide, and UVGI are all reasonable options. In particular, UVGI has been demonstrated to be effective for FFR-DR and has been successfully employed at our institution. It should be emphasized that each specific brand of FFR is unique and UVGI may not be effective for all. Strap degradation associated with repeated don/doff and UVGI cycles and consequent fit dysfunction are chief limitations for UVGI FFR decontamination. However, its ability to scale to large tertiary care institutions due 
to its fast throughput time as well as its use of already-available technology (many hospitals us UVGI for environmental decontamination) make it an attractive strategy. It should also be noted that FFR decontamination and reuse should only be used in emergency situations and as soon as an adequate supply of FFRs can be secured, manufacturer recommendations regarding use and decontamination should be followed. Institutions interested in more durable forms of respiratory protection are encouraged to explore the use of elastomeric respirators or PAPRs. However, with proper implementation and procedures as well as adequate risk-benefit assessment, N95 FFR-DR, particularly using UVGI, can maximize scarce FFR resources in the setting of an emergency. More work will need to be continued within this field to ensure that institutions are better prepared for future outbreaks.

\section{Acknowledgements}

We wish to thank all of the courageous medical professionals who have been involved in the compassionate care of persons infected with SARS CoV-2 at our institution and around the globe.

\section{Declaration}

Human and animal rights and informed consent

This article does not contain any studies with human or animal subjects performed by any of the authors.

\section{Conflict of interest}

Mark P. Ridder, Katie D. Paladino, John J. Lowe, and Mark E. Rupp declare that they have no conflicts of interest.

\section{References and Recommended Reading}

Papers of particular interest, published recently, have been highlighted as:

- Of importance

- Of major importance

1. Bartoszko JJ, Farooqi MAM, Alhazzani W, Loeb M. Medical masks vs N95 respirators for preventing COVID-19 in healthcare workers: a systematic review and meta-analysis of randomized trials. Influenza Other Respir Viruses. 2020;14(4):365-73. https://doi. org/10.1111/irv.12745.

2.• Mills D, Harnish DA, Lawrence C, Sandoval-Powers M, Heimbuch BK. Ultraviolet germicidal irradiation of influenza-contaminated N95 filtering facepiece respirators. Am J Infect Control. 2018;46(7):e49-55. https://doi.org/10.1016/j.ajic.2018.02.018.

Comprehensive testing of effectiveness of UVGI decontamination of 15 N95 FFR models when contaminated with influenza and soiling agent.

3. $\quad$ Centers for Disease Control and Prevention. Implementing filtering facepiece respirator (FFR) reuse, including reuse after decontamination, when there are known shortages of N95 respirators. 2020; Available at: https://www.cdc.gov/coronavirus/2019-ncov/ hcp/ppe-strategy/decontamination-reuse-respirators. html. Accessed 11/10, 2020.

CDC strategy for optimizing supply of N95 FFRs in crisis situations.

4. Kapust PJ. Enforcement guidance for respiratory protection and the N95 shortage due to the coronavirus disease 2019 (COVID-19) pandemic 2020; Available at: https://www.osha.gov/memos/2020-04-03/ 
enforcement-guidance-respiratory-protection-andn95-shortage-due-coronavirus. Accessed 11/10, 2020.

5. Emergency Use Authorization (EUA) information, and list of all current EUAs. 2020; Available at: https://www. fda.gov/emergency-preparedness-and-response/mcmlegal-regulatory-and-policy-framework/emergency-useauthorization\#covidppe. Accessed 11/10, 2020.

6. Viscusi DJ, Bergman MS, Eimer BC, Shaffer RE. Evaluation of five decontamination methods for filtering facepiece respirators. Ann Occup Hyg. 2009;53(8):815-27. https://doi.org/10.1093/annhyg/ mep070.

7. Lore MB, Heimbuch BK, Brown TL, Wander JD, Hinrichs $\mathrm{SH}$. Effectiveness of three decontamination treatments against influenza virus applied to filtering facepiece respirators. Ann Occup Hyg. 2012;56(1):92-101.

8. Pascoe MJ, Robertson A, Crayford A, Durand E, Steer J, Castelli A, et al. Dry heat and microwave-generated steam protocols for the rapid decontamination of respiratory personal protective equipment in response to COVID-19-related shortages. J Hosp Infect. 2020;106(1):10-9. https://doi.org/10.1016/j.jhin. 2020.07.008.

9. Widmer AF, Richner G. Proposal for a EN 149 acceptable reprocessing method for FFP2 respirators in times of severe shortage. Antimicrob Resist Infect Control. 2020;9(1):88.

$10 . \bullet \quad$ Heimbuch B HD. Research to mitigate a shortage of respiratory protection devices during public health emergencies. Report prepared for Food and Drug Administration. Contract

number:HHSF223201400158C. 2019; Available at: https://www.ara.com/wp-content/uploads/ MitigateShortageofRespiratoryProtectionDevices_3. pdf. Accessed 11/28, 2020.

Comprehensive summary of research regarding effectiveness of UVGI decontamination of N95 FFRs.

11. Heimbuch BK, Kinney K, Lumley AE, Harnish DA, Bergman M, Wander JD. Cleaning of filtering facepiece respirators contaminated with mucin and Staphylococcus aureus. Am J Infect Control. 2014;42(3):26570. https://doi.org/10.1016/j.ajic.2013.09.014.

12. NPPTL Respirator Assessments to Support the COVID19 Response. 2020; Available at: https://www.cdc.gov/ niosh/npptl/respirators/testing/default.html. Accessed 11/10, 2020, DOI: https://doi.org/10.1097/QAI. 0000000000002609.

13. Lowe JJ, Paladino KD, Farke JD, Boulter K, Cawcutt K, Emodi M, et al. N95 Filtering facepiece respirator ultraviolet germicidal irradiation (UVGI) process for decontamination and reuse. 2020; Available at: https:// www.nebraskamed.com/sites/default/files/ documents/covid-19/n-95-decon-process.pdf.
Accessed October 19, 2020, DOI: https://doi.org/10. 11604/pamj.supp.2020.35.2.22794.

14. Viscusi DJ, Bergman MS, Novak DA, Faulkner KA, Palmiero A, Powell J, et al. Impact of three biological decontamination methods on filtering facepiece respirator fit, odor, comfort, and donning ease. J Occup Environ Hyg. 2011;8(7):426-36. https://doi.org/10. 1080/15459624.2011.585927.

15. Bergman MS, Viscusi DJ, Heimbuch BK, Wander JD, Sambol AR, Shaffer RE. Evaluation of multiple (3Cycle) decontamination processing for filtering facepiece respirators. J Eng Fibers Fabr 2010 12/01; 2020/11;5(4):155892501000500405.

16. Lindsley WG, Martin SB Jr, Thewlis RE, Sarkisian K, Nwoko JO, Mead KR, et al. Effects of ultraviolet germicidal irradiation (UVGI) on N95 respirator filtration performance and structural integrity. J Occup Environ Hyg. 2015;12(8):509-17. https://doi.org/10.1080/ 15459624.2015.1018518.

17. Viscusi DJ, King WP, Shaffer RE. Effect of decontamination on the filtration efficiency of two filtering facepiece respirator models. J Int Soc Respir Prot. 2007;24(3/4):93.

18. O'Hearn K, Gertsman S, Sampson M, Webster R, Tsampalieros A, Ng R, et al. Decontaminating N95 and SN95 masks with ultraviolet germicidal irradiation does not impair mask efficacy and safety. J Hosp Infect. 2020;106(1):163-75. https://doi.org/10.1016/j.jhin. 2020.07.014.

19. Bergman MS, Viscusi DJ, Zhuang Z, Palmiero AJ, Powell JB, Shaffer RE. Impact of multiple consecutive donnings on filtering facepiece respirator fit. Am J Infect Control. 2012;40(4):375-80. https://doi.org/10.1016/ j.ajic.2011.05.003.

20. Degesys NF, Wang RC, Kwan E, Fahimi J, Noble JA, Raven MC. Correlation between N95 extended use and reuse and fit failure in an emergency department. JAMA. 2020;324(1):94-6. https://doi.org/10.1001/ jama.2020.9843.

21. Fisher EM, Shaffer RE. Considerations for recommending extended use and limited reuse of filtering facepiece respirators in health care settings. J Occup Environ Hyg. 2014;11(8):D115-28. https:// doi.org/10.1080/15459624.2014.902954.

\section{Publisher's Note}

Springer Nature remains neutral with regard to jurisdictional claims in published maps and institutional affiliations. 